\title{
Dynamics of Structure Formation in Model Membranes and in Adsorbed Layers
}

\author{
H. MöhWald, ${ }^{*}$ U. HöHNe, and P. Quint \\ Universität Mainz, Institut für Physikal, Chemie, \\ Welder-Weg 11, D6500 Mainz, Germany \\ (Received November 26, 1990)
}

\begin{abstract}
Unconventional and new ways to prepare ultrathin films with defined lateral distribution of components are described. These methods make use of two different principles: (1) Films with two coexisting phases are prepared and an active component preferentially partitions into one of the phases. Domain formation and interdomain distance largely depend on the kinetics of nucleation and growth and can thus be controlled. This is demonstrated by fluorescence microscopy with monolayers on water surfaces as well as with monolayers formed by co-adsorption of a fatty acid and a dye on silicon.

(2) The support is structured by conventional techniques, in our case by photolithography. Adsorption or desorption then occur preferentially on one of the two types of surfaces. It is demonstrated by optical microscopic techniques that this way optically active molecules can be arranged purposefully with a lateral resolution better than $0.5 \mu \mathrm{m}$ and also inorganic particles can be prepared and deposited with high precision.
\end{abstract}

Key words 2D order/Organic Films / Structure Formation/Lipid Monolayers/Surfactant Films/ Microscopy.

Experiments and Discussion: Laterally structuring an ultrathin film is highly important for any technical application. It can principally be achieved by conventional lithographic techniques, but it is also possible to use natural principles of self-organization. The latter is demonstrated within this work. We will thus elaborate on some of the basic principles and provide 
experimental data on their use. It turns out that this work not only serves futuristic technical goals but also an understanding of interactions that may be important for the function of biological membranes.

One of the basic principles is most clearly demonstrated by inspecting a lipid monolayer at the air/water interface by fluorescence microscopy (Fig. 1). Lateral molecular density and surface pressure are established such that the monolayer is in coexistence of a fluid and a more ordered phase, and a dye probe is inserted that, like most impurities, preferentially partitions into the disordered phase.

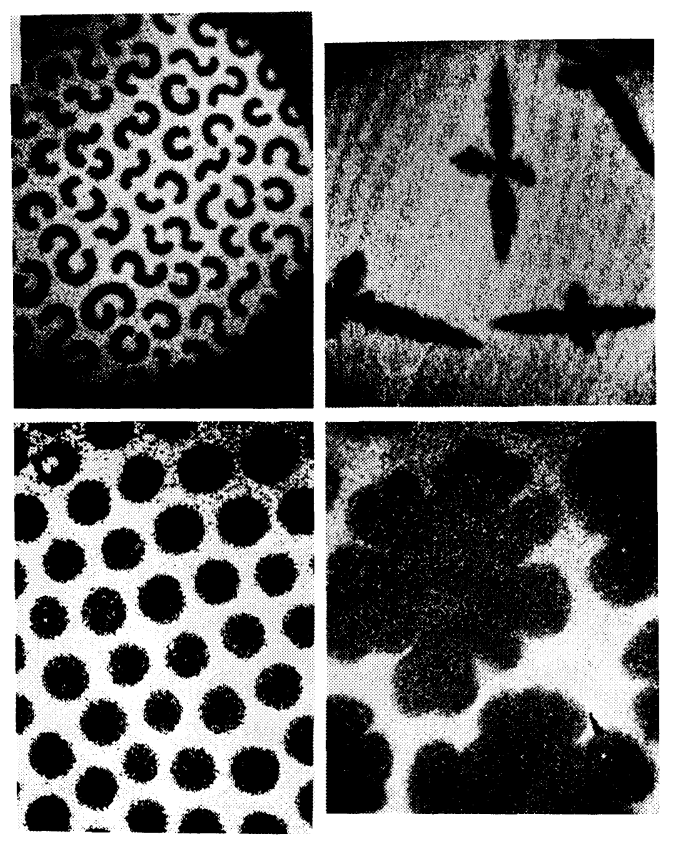

Fig.1: Fluorescence micrographs of lipid monolayers, containing about 1 mole\% of a dye probe, in the coexistence range of a fluid and a more ordered phase. Upper left: Acidic phosphoplipid at high $\mathrm{pH}$ (11.5) with 1 mole\% cholesterol. Lower left: Film as above at high $\mathrm{pH}$, but with $100 \mathrm{mM} \mathrm{NaCl}, 1 \mathrm{mM}$ $\mathrm{CaCl}_{2}$ and without cholesterol.

Upper right: Diacetylenic lipid. Lower right: Acidic phospholipid at pH 5.5.

The images in the left row of Fig. 1 clearly show that a periodic dye distribution can be achieved this way. The formation of ordered phase domains of uniform size, shape and distance can be understood as a local equilibrium feature from an interplay of line tension and electrostatic forces.1,2 The latter result from an excess density of the vertical dipole moment connected with the surfactant and oriented at the interface, comparing ordered and less ordered phases. This long range force principally limits the domain size, favours non-compact shapes and shape instabilities and effects a strong repulsion between domains which for uniform sizes leads to a Wigner lattice. It is opposed by line tension, the energy per length to 
prepare a boundary between two phases, which in turn favours compact shapes.

It can be shown theoretically that as a result hexagonal or lamellar superstructures may be encountered 1 and the dimensions of structures may be varied via their dependence on these forces through subphase ionic conditions 3,4, type of lipid and surfactant impurities. 5 In praxis, however, the domain shapes may be only equilibrium features, 6 in contrast to their size and superlattice dimensions. ${ }^{7}$ The latter depend on the nucleation kinetics: There is hardly any splitting or merging of domains. Hence the number of domains formed during the nucleation period remains constant on compressing the monolayer from the fluid into the ordered phase. 8 Thus to control the lateral molecular distribution one has to understand the kinetics of domain formation. An experiment serving this understanding is given in Fig. 2.

The expanded monolayer is quickly compressed into the phase coexistence range. Nucleation and growth of single domains can then be observed under favorable conditions as well as the time dependence of surface pressure and dye distribution near the domain boundary. 9 All observations can be understood within the classical frame work of constitutional supercooling: During crystallization impurities are squeezed from the ordered phase into the disordered environment. There they increase the surface pressure to enable further crystallization. The latter can only continue if the local impurity enrichment is removed by diffusion. Hence crystallization is diffusion limited, and this limitation also favours noncompact shape: Increasing the boundary length facilitates removal of impurities. Computer simulations of diffusion limited aggregation (DLA) processes have yielded self-similar structures as those of Fig. 2 with a fractal dimension of 1.5.10 This is indeed also derived from a quantitative analysis of the fluorescence micrographs measured shortly after fast compression. 9 At later times the influence of line tension can be observed as a smoothing of domain boundaries. 5 

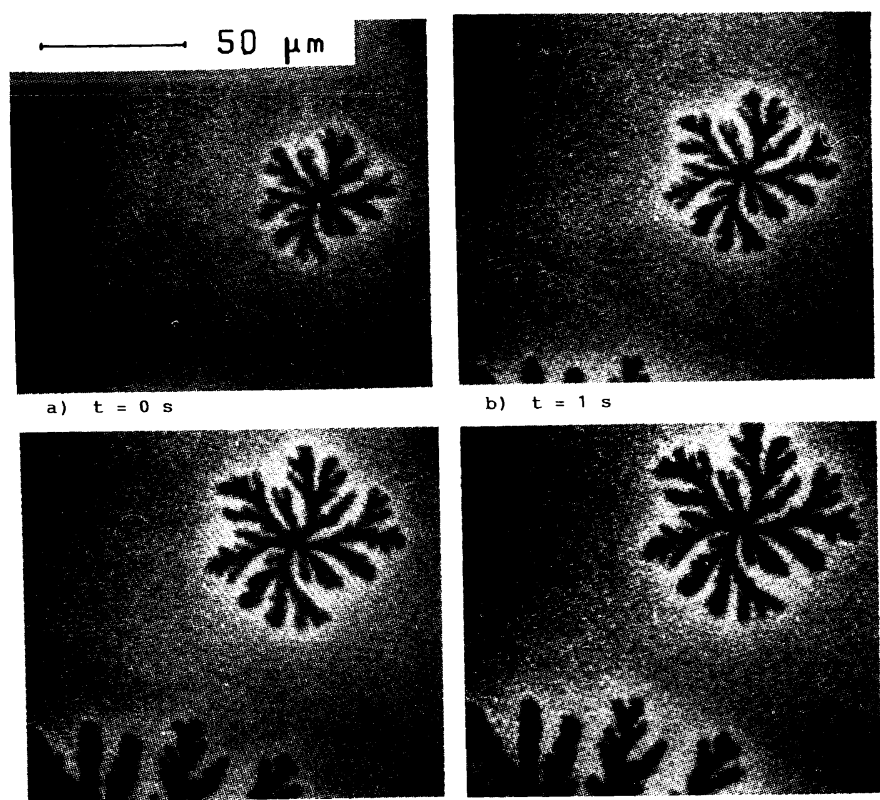

b) $t=1 s$

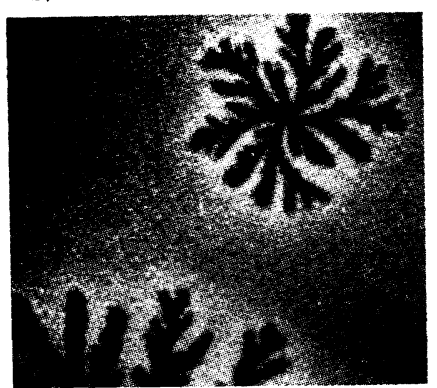

c) $t=2 s$
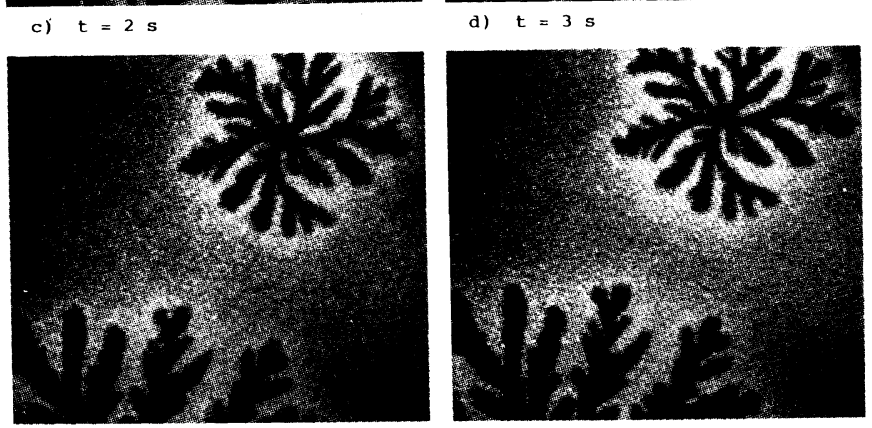

e) $t=4 \mathrm{~s}$

f) $t=5 s$

Fig.2: Fluorescence micrograph of a phospholipid monolayer after stepwise barrier compression and then fixing of the barrier. The time after compression is indicated.

Considering the dye as a typical impurity one can measure its distribution via quantitative image analysis and also measure the relation between impurity concentration and increase of transition pressure. With these data pressure relaxation and domain growth as well as dye distribution could be modeled in almost quantitative agreement with experimental results. ${ }^{5}$

The influence of impurities on reduction of line tension can also be used to control the kinetics of nucleation as well as the annealing of sharp boundaries 
of domains. Thus at higher dye content more domains with sharper boundary edges could be observed.

In most cases of phospholipid or fatty acid monolayers the domain shape is not influenced by apparently too weakly anisotropic crystal forces. However, there are also notable examples of polymerizable lipids 11 , amphiphilic cyanine dyes 12 and branched chain phospholipids 13 where the anisotropy of the chain lattice is expressed in the domain morphology (see upper right of Fig. 1).

It is also remarkable that the domains could be transferred on solid support without grossly altering the domain morphology. 4 In addition the excess charge density of a domain could be used to enable crystallization of a water soluble dye with opposite charge. Thus the surface layer could be replicated. 14

The unique feature governing structure formation in monolayers on water, two-dimensional transport processes and anisotropic molecular alignment at an interface may also hold for monolayers adsorbing on solid surfaces. In order to observe these features the adsorbing molecule has to be mobile which can be also achieved via reversible ad/desorption. This condition is fulfilled for fatty acids adsorbing from organic solvent, and an experiment making use of it is presented in Fig. 3. In this case a fatty acid and a fluorescent dye are coadsorbed on a slide which is then removed from the solution and observed through a fluorescence microscope. One thus measures the dye distribution and the similarity with the fractal structure of Fig. 2 is obvious. Apparently the adsorption of the fatty acid is followed by nucleation and growth of an ordered structure that excludes the dye. The nucleation density surely depends on the type of lipid and preparation conditions, and this dependence is presently under study as well as the time dependence of domain formation. In some cases one can also observe a dye halo around a domain indicating that the dye excluded from the ordered phase remains at the interface. Hence self organization during adsorption resembles that the air/water interface, but it is to be expected that the nucleation density may vary over orders ofmagnitude, and we have by chance encountered situations where the domains are sufficiently large to be observable by fluorescence microscopy. 

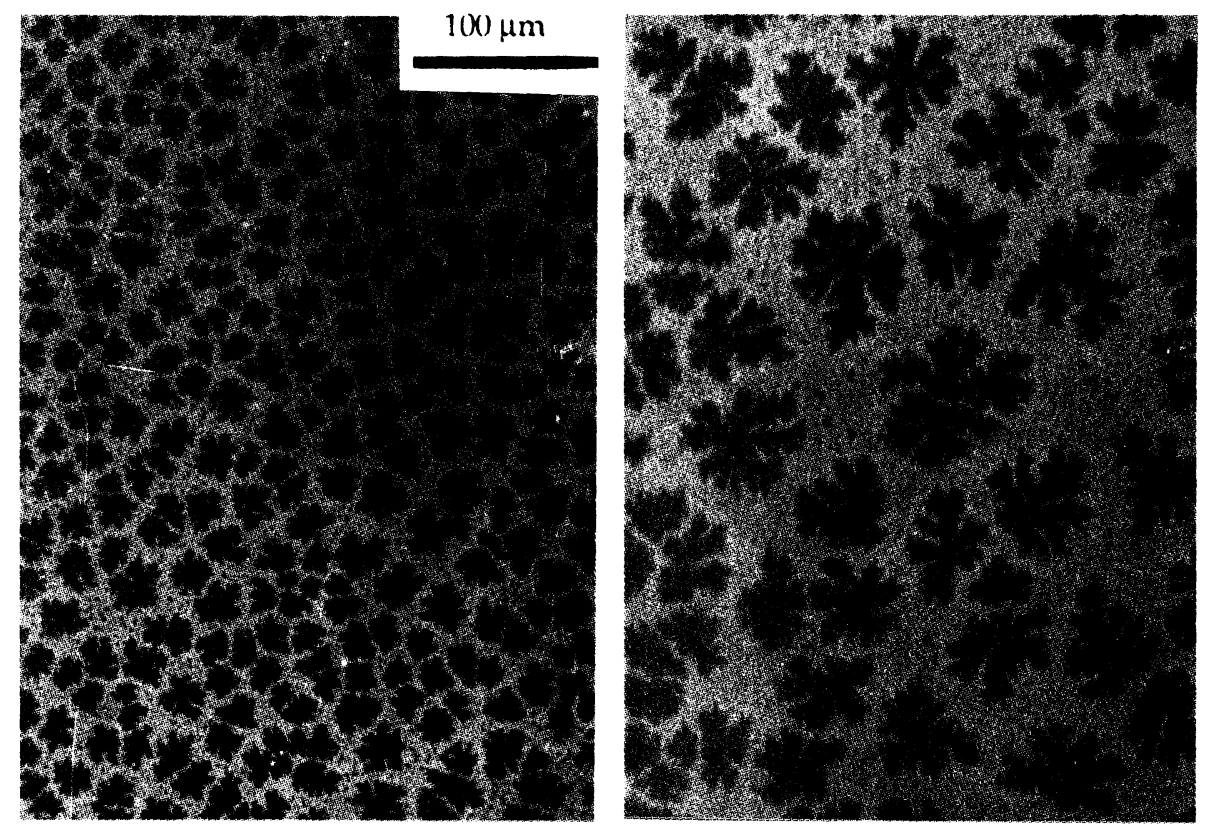

Fig.3: Fluorescence micrograph of a glass slide after 3-4 minutes of co-adsorption of arachidic acid (left) or behenic acid (right) and dye-labeled undecanoic acid.

Solution: Bicyclohexane/chloroform 9:1, $5^{*} 10^{-4} \mathrm{M}$ surfactant concentration, $\leq 10^{-6} \mathrm{M}$ dye concentration.

A quantitatively different mode of preparing a lateral structure results from suitable treatment of the support before deposition of an ultrathin film. We have recently shown that a LB film can be completely removed by UV light from a $\mathrm{SiO}_{2}$ support. 15,16 This also holds for a silane monolayer, and illumination of the support via a mesh therefore effects neighbouring hydrophilic and hydrophobic surface areas. Immersing the surface into a solution containing an amphiphilic dye then enables adsorption of the dye at the hydrophilic surface. This can be visualized by fluorescence microscopy (Fig. 4a). Quantitative analysis of the images then shows that the edges are sharper than $0.5 \mu \mathrm{m}$, the resolution being limited by the quality of UV imaging (Fig. 4b). 

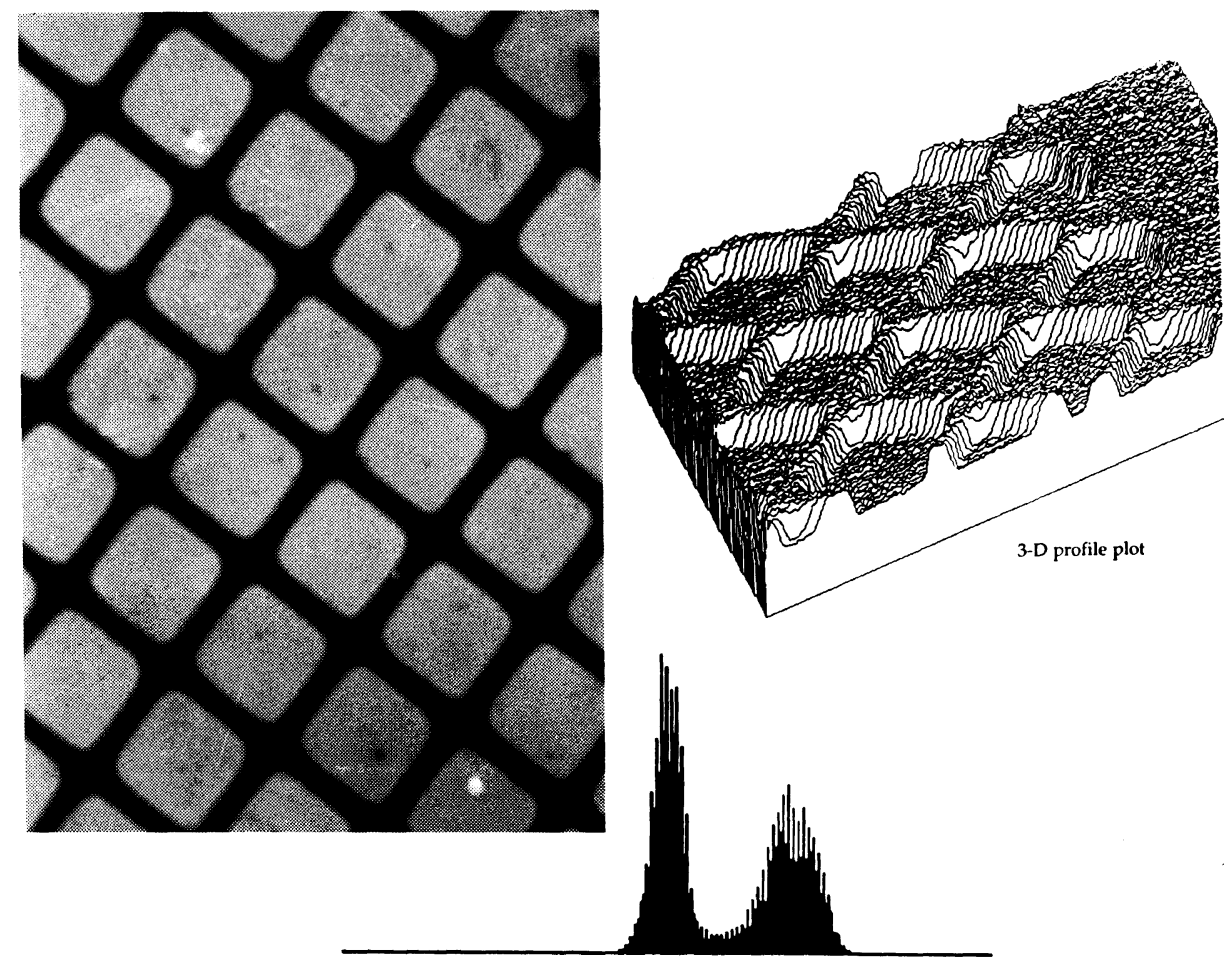

Distribution of gray values

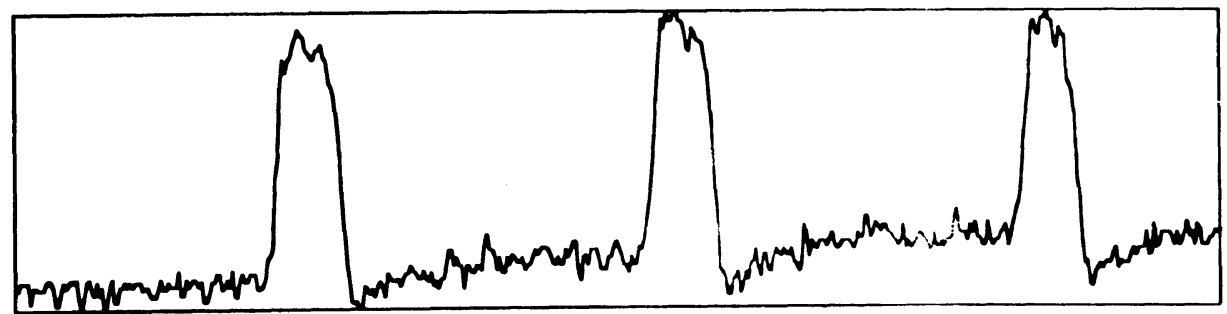

Fig.4: Upper left: Fluorescence micrograph of a silanized glass slide that was UV illuminated through a mesh with $40 \mu \mathrm{m}$ distance and then immersed in a solution containing the dye of Fig. 3.

Upper right: 3D profile plot of the lateral intensity, distribution of pixels as a function of intensity.

Bottom: Density distribution along a line through the image.

The possibility to prepare a surface with defined hydrophilic and hydrophobic areas and sharp boundary lines can also be used to arrange inorganic particles in a predetermined way. An example on this is given in Fig. 5. 


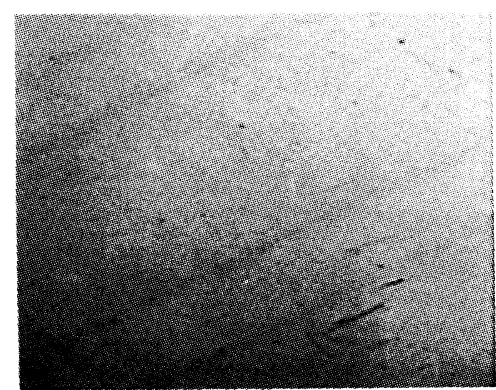

$\mathrm{T}=25^{\circ} \mathrm{C}$

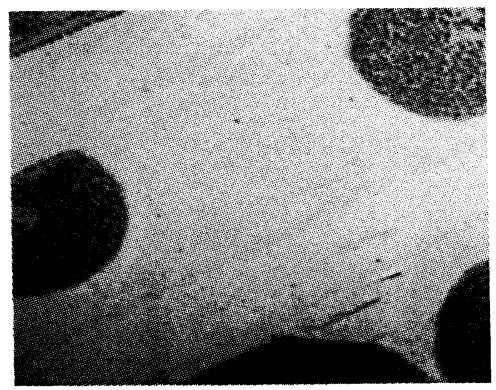

$\mathrm{T}=117^{\circ} \mathrm{C}$

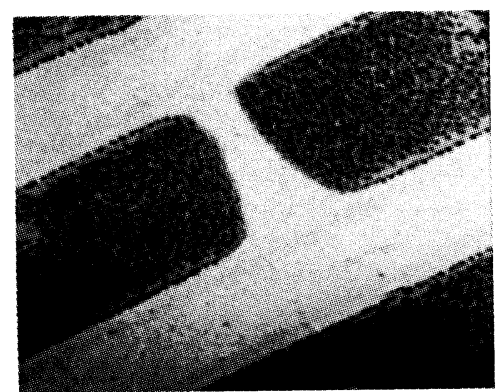

$\mathrm{T}=119^{\circ} \mathrm{C}$

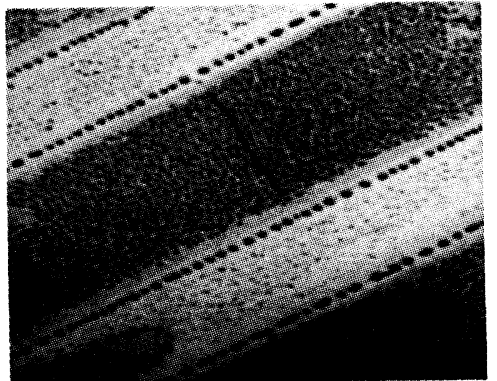

$\mathrm{T}=130^{\circ} \mathrm{C}$

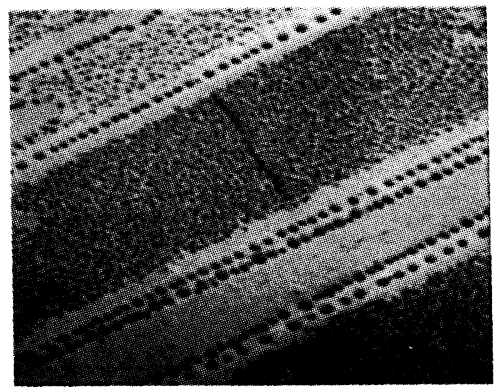

$\mathrm{T}=136^{\circ} \mathrm{C}$

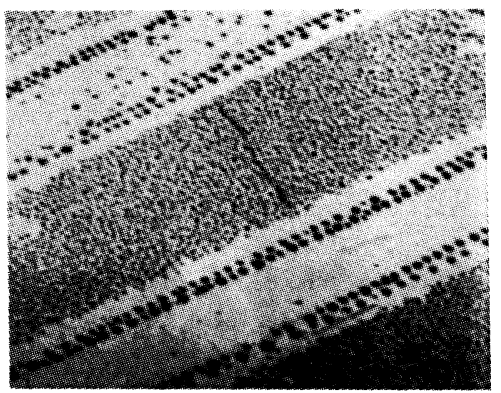

$\mathrm{T}=148^{\circ} \mathrm{C}$

Fig.5: Nomarsky photomicrograph of a Si wafer, silanized, then illuminated through a stripe mask and coated with 10 monolayers of $\mathrm{Pb}^{2}+$ stearate at different temperatures. The surface area not photodesorbed and hence coated by the silane exhibits higher film stability. 
A silanized Si wafer with part of it photodesorbed through a mask is coated with a $\mathrm{LB}$ film of a fatty acid salt (in this case $\mathrm{Pb}^{2+}$ stearate). Heating the sample the silane remains stable whereas the fatty acid forms a fluid film as judged from small angle X-ray scattering. 17 It then forms droplets, the organic component desorbs and the inorganic fraction remains as proved from ESCA analysis. 18 The characteristic temperatures and the detailed process depend among others on the support, and this can be seen in the Nomarsky micrographs of Fig. 5. In this example the film on the hydrophilic area is less stable and one can see first the rupture of the film and break up into droplets at this part. At higher temperatures the LB film on the hydrophobic area also breaks into droplets and one can see this as a moving front of dewetting. In the latter case larger droplets moving to the side of the stripe-like areas are encountered and the subsequent change in contrast is due to evaporation leaving behind the particle of the inorganic salt. These are distributed along stripes that were previously selected by photodesorption of the silane from the wafer.

a) Coexistence Ordered-Disordered Phases

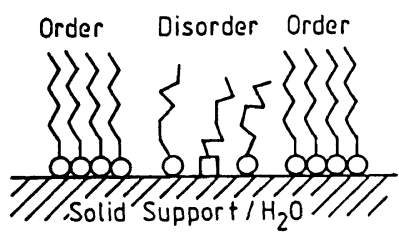

Fig.6: Sketch of the different mechanisms leading to lateral structure in thin films.

b) Support Structuring before Film Depos.

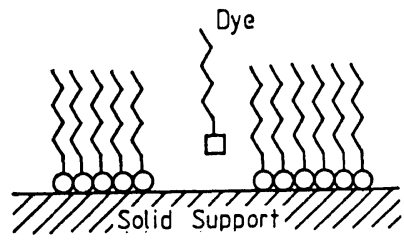

a.) Phase coexistence with an active component in one of the phases.

b.) Removing a fraction of the monolayer by lithographic techniques to enable deposition of another surfactant or to ease desorption of

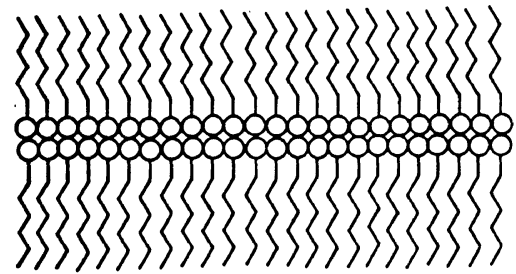
multilayers on top of the surface.

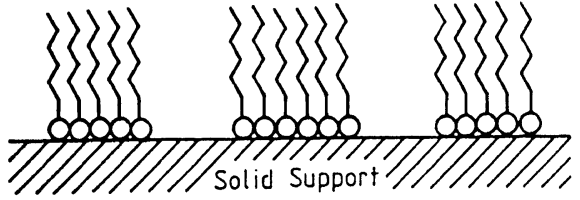


In conclusion we have demonstrated different ways to prepare laterally heterogeneous distributions of components of a thin film. Their principles are sketched in Fig. 6.

In Fig. $6 a$ the fact is used that one component is less soluble in one of the coexisting phases. These phases can in turn be arranged periodically making use of electrostatic forces arising from the interfacial nature and controlling the kinetics of nucleation and growth. In the second mode discussed (Fig. 6b) the surface is structured before film deposition by adsorption or by the LB technique. Then either adsorption is laterally selective or we can use the dependence of film stability on support to develop defined later molecular or atomic distributions. These latter processes are quantitatively not yet well characterized and described, but they are clearly near the front of present interfacial research exemplified with key words like cooperative adsorption and dewetting.

ACKNOWLEDGEMENT: We have reported in the first part on work at the air/water interface that was already published together with members of our group. In the second part we have described presently ongoing work which is supported by the Bundesministerium für Forschung und Technologie (BMFT) and by the Fonds der Chem. Industrie.

\section{Literature:}

1. D.Andelman, F.Brochard, J.F.Joanny, J.Chem.Phys. 86, 3673 (1987)

2. A.Fischer, M.Lösche, H.Möhwald, E.Sackmann, J.Phys.Lett. 45, 785 (1984)

3. C.A.Helm, L.Laxhuber, M.Lösche, H.Möhwald, Colloid Polym.Sci. 264, 46 (1986)

4. M.Lösche, C.A.Helm, H.D.Mattes, H.Möhwald, Thin Solid Films 133, 51 (1985)

5. A.Miller, H.Möhwald, J.Chem. Phys. 86, 4258, (1987)

6. D.J.Keller, H.M.McConnell, V.T.Moy, J.Phys.Chem.90, 3211 (1986)

7. C.A.Helm, H.Möhwald, J.Phys.Chem. 92, 1262 (1988)

8. M.Lösche, H.P.Duve, H.Möhwald, J.Coll. Interf.Sci. 126, 432 (1988)

9. A.Miller, W.Knoll, H.Möhwald, Phys.Rev.Lett. 56, 2633 (1986)

10. J.Nittman, H.E.Stanley, Nature 321, 663 (1986)

11. H.D.Göbel, H.Gaub, H.Möhwald, Chem. Phys. Lett. 138, 441 (1987)

12. C.Duschl, M.Lösche, A.Miller, A.Fischer, H.Möhwald, W.Knoll, Thin Solid Films 133, 65 (1985)

13. A.Dietrich, H.Möhwald, W.Rettig, G.Brezesinski, Langmuir, in press

14. S.Kirstein, H.Möhwald, M.Shimomura, Chem.Phys.Lett. 154, 303 (1989)

15. L.A.Laxhuber, H.Möhwald, Langmuir 3, 837 (1987)

16. P.Tippmann-Krayer, L.A.Laxhuber, H.Möhwald, Thin Solid Films, 159, 387 (1988)

17. P.Tippmann-Krayer, Y.Lvov, H.Möhwald, subm. to J.Phys.Chem.

18. P.Tippmann-Krayer, W.Meisel, H.Möhwald, Adv.Mat. (1990) in press 